\title{
Perimetric Distributed UV Reactor and Its Validation and the Decontamination of Fresh Broccolis
}

\author{
Bruno Pereira de Oliveira*, Shirlei Lara, Daniel Chianfrone, Kate Cristina Blanco, \\ Vanderlei Salvador Bagnato
}

São Carlos Institute of Physics, University of São Paulo, São Carlos, Brazil

Email address:

Pereirabrunnol@gmail.com (B. P. de Oliveira), vander@ifsc.usp.br (V. S. Bagnato)

${ }^{*}$ Corresponding author

\section{To cite this article:}

Bruno Pereira de Oliveira, Shirlei Lara, Daniel Chianfrone, Kate Cristina Blanco, Vanderlei Salvador Bagnato. Perimetric Distributed UV Reactor and Its Validation and the Decontamination of Fresh Broccolis. American Journal of Applied Chemistry.

Vol. 7, No. 6, 2019, pp. 161-167. doi: 10.11648/j.ajac.20190706.12

Received: October 16, 2019; Accepted: November 25, 2019; Published: December 2, 2019

\begin{abstract}
The ultraviolet (UV) irradiation as a non-thermal processing technique for microbial decontamination of food (MDF) has been the gainer in many variations after the inclusion of UV light as an alternative for MDF by the US FDA. However the lasts years increase the application of the UV light in food, water and pharmaceutical utilization. In this report, we describe a new type of reactor, where the UV emitters are parametrically distributed for decontaminating fresh broccolis. We described the constructed reactor and its characterization with the validation of the system with controlled contaminated broccolis. The overall liquid was contamined with $10^{5} \mathrm{UFC} / \mathrm{mL}$ E. coli operating with a flow rate of $80 \mathrm{~L} / \mathrm{min}$ in $30 \mathrm{~L}$ and six lamps in the reactor and the collection of samples in intervals of $25 \mathrm{~min}$. The $E$. coli used in this experiment was eliminated in 99,99\% The intensity of UVC light distributed in the internal part of the reactor is practically homogeneous due to the developed geometry. The kinetics of microbial death presented no great influence on this variation. That is, any volume of water contained in the process can be decontaminated. A relation between UV and the flow rate was stablished. The system demonstrated its capacity in inactivating the microorganism.
\end{abstract}

Keywords: Reactor, Foods, Innovation, Decontamination

\section{Introduction}

The use of ultraviolet (UV) radiation as a non-thermal processing technique for microbial decontamination of food (MDF) has been the gainer in many variations after the inclusion of UV light as an alternative for MDF by the US FDA. It turns out that directly use of UV light on the food is not always delivered due to geometry prevent light to reach all necessary parts or UV light incident on pigmented molecules may cause photobleaching with color modification [1].

For the in nature food like fresh vegetables and others, the thermal inactivation of microorganisms is not always the best choice for decontamination. Food washing with acidic water is the most employed alternative. In that case, vast amounts of water are necessary to reach the recommended reduction of the pathogens quantity is either economic environmental problem when the acid water is discharged [2-4].
An attractive alternative for this situation is the use of closed circuit water circulation with a stage of microbial inactivation using an efficient UV reactor. The water may remove the microorganisms that are inactivated followed by the return of the water to the circuit. That solution has many advantages: the volume used water is considerable decreased and the use of acid considerable reduced; in addition, the return of water to the environment causes no damage or needs a previous to discharge processing. For that to work, efficient UV reactor, dealing with charge water volume is necessary [2, $5,6]$.

In this report, we describe a new type of reactor, where the UV emitters are parametrically distributed for decontaminating fresh broccolis. The decontamination of fresh broccolis, without thermal or high acid solution, is a challenge due to the complex geometry of the target for decontamination. If a technique to show high efficiency in decontaminating broccolis, can be possibly employed in many 
other application $[3,7]$.

We start our report, describing the constructed reactor, and its characterization. After, the validation of the system with controlled contaminated broccolis is described. Conditions of operation and basic understanding of the inactivation kinetics are presented and discussed.

\section{Materials and Methods}

\subsection{Manufacturing Reactor of the $U V-C$}

Table is a follow: Firstly, the construction of a convergent UV-C reactor with aluminum was necessary. A quartz circulation system utilized the tube and connection.

Table 1. Materials applied for construction the UV reactor.

\begin{tabular}{lll}
\hline Total & Diameter (pol.) & Material \\
\hline Globe valves & $1 / 2 "$ & Galvanized \\
Flow meter & $1 / 2 "$ & PVC \\
Quartz tube & $2 "-1 / 2 "$ & Galvanized/PVC \\
Structure & Variable & Iron \\
Union & $1 / 2 "$ & PVC \\
UV lamp & $1 / 2 "$ & Quartz/metal \\
\hline
\end{tabular}

After step of the construction, the verification of the compliance aspect and certificated the flow water were performed, not have the leakage between connection and tubes. To this, one testing volume was insert to $50 \mathrm{~L}$ of water in environment temperature and circulated by 2 hours in the all system.

Consequently, the pieces of vegetable that simulated the lixiviation were inserted. However, the generation turbulence in the washing tank presented problems with sediments of the vegetable and entrance in the pump.

Because this point and to promote a security and integrality of experimental design the gage on the process control possibility of the problem in the upstream pump (Figure 1).

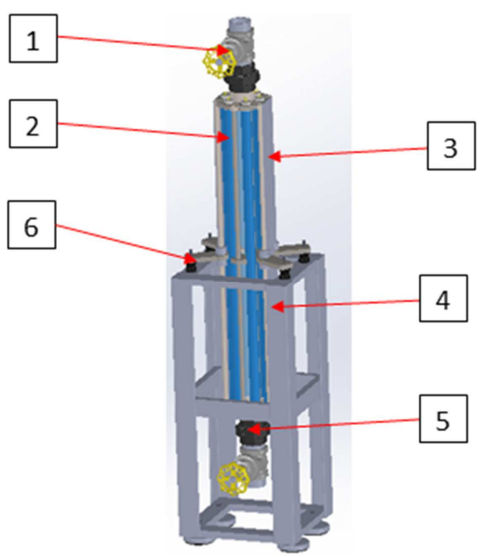

Figure 1. Schematic design of the reactor; 1-) Valve to control the downstream; 2-) UV light of the $30 \mathrm{~W}$ Osram; 3-) Support of the lamp; 4-) Basic structure of the system; 5-) Valve to upstream.

\subsection{Microbiological Validation}

Microbial inactivation tests were performed with $E$. coli ATCC 25922 belongs to the culture collection of the
Biophotonics Laboratory of the São Carlos Institute of Physics (SP, Brazil). A broccoli contamination was applied in samples with weight approximately $9,95 \mathrm{~g}$ of broccoli in the $50 \mathrm{~mL}$ of the microorganism solution for each experimental system; a vegetable was immersed in solution with $10^{8} \mathrm{CFU} / \mathrm{mL}$ of the $E$. coli by agitation for 2 minutes [8]. E. coli in suspension 10-5 $(\mathrm{CFU} / \mathrm{mL})$ were inoculated in tank for minutes under UVC. After procedural time intervals, aliquots $(100 \mu \mathrm{L})$ were diluted in $10^{-1}$ to $10^{-6}$ in PBS and it takes to determine colony-forming units (CFU). Table is a follows: The test time and analyzing.

Table 2. Experimental date to microbiological analyzing.

\begin{tabular}{ll}
\hline Variables & date \\
\hline Flow & $20 / 40 / 60 / 80(\mathrm{~L} / \mathrm{min})$ \\
Analyze water & yes \\
Analyze broccoli & yes \\
Power of lamp/unit & $30 \mathrm{~W}$ \\
Time analyze & $10 / 30 / 50 / 70 / 100 \mathrm{~min}$ \\
\hline
\end{tabular}

\subsubsection{Optical Analyzing and Characterization}

To characterize the luminous intensity each lamp utilized the sensor LM-2 with range of $250-400 \mathrm{~nm}$, power lamp is $30 \mathrm{~mW}$ and resolution is $1 \mathrm{nW}$ and uncertainty of $8 \%$. (Figure 2).
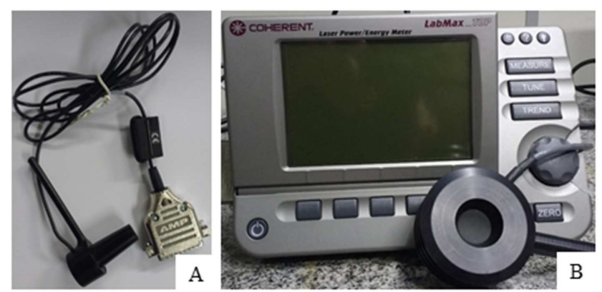

Figure 2. Equipment to characterized of luminous light of the lamp; A-) COHERENT sensor LM-2 UV of the series 0482116R, to measures on track on ultraviolet light; B-) Wattage meters the mark COHERENT model LABMAX-TOP.

For mechanical project, the inventor software Tracepro was utilized for optical rays analysis. Whereby defined the emission fonts for each lamps and the irradiation is radiometric units and angular distribution was Lambertian with irradiance $1 \mathrm{~W} / \mathrm{cm}^{2}$ and $254 \mathrm{~nm}$ wavelength and range 10 -1000 rays.

\section{Results and Discussion}

\subsection{Perimetric Emitters $U$ V-reactor}

Ultraviolet (UV) light for disinfection of water and wasterwater is becoming common in many situations and therefore a frequently employed alternative. UV-C, B, A and combination of them are alternative for use. Cylindrical Hg-lamps is the most economically viable light source for those applications. Most of the known geometry are composed of an axial lamp, surrounded by a camera in which fluid circulated. This geometry is normally referred as axial emitters (Figure 3b).

It has the advantage that all the light is incident on the fluid as indicated in Figure 3a. The system, however, the fast 
intensity of light decrease as we radially moves the distance of the emitter. Fluid in contact with the inner part of the circulating chamber receives move UV light when compared with the most outside part of fluid (Figure 1b) [9-13].

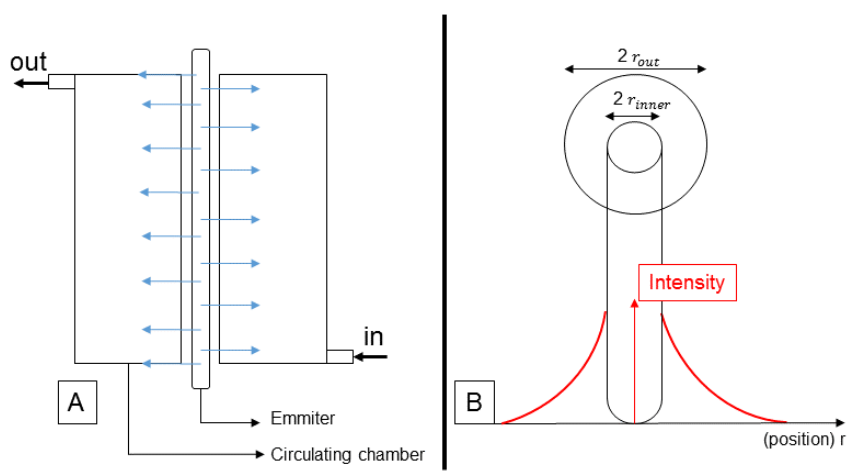

Figure 3. A-) Design of the reactor with radial emitter; $B$-) Profile of the UV light in the reactor.

In the above referred axial geometry, the ratio between the most inner surface to the most out surface, light intensity goes naturally (without considering absorption) as $\frac{I_{\text {out }}}{I_{\text {inner }}}=\frac{r_{\text {inner }}}{r_{\text {out }}}$ having a change to be a quite small ratio if the volume of the circulating chamber is large $\left(\mathrm{r}_{\text {out }}>>\mathrm{r}_{\text {inner }}\right)$.

In that situation, it is hard to assure a constant UV expose dose to all the circulating fluid if only a few passages take place. An alternative way is using the perimetric distribution of light sources. In this geometry, the light emitters are not at the center, rather around the chambers in a perimetric distribution.

If we consider a certain numbers $\mathrm{N}$ of emitters, parametrically distributed as illustrated in Figure 2a considering $r_{0}$ the radios of each emitter and $I_{0}$ the correspondent surface light intensity, the light intensity at any position $(\mathrm{x}, \mathrm{y})$, is given by the sum of all contributions as indicated in figure $2 \mathrm{~b}\left(\mathrm{x}_{\mathrm{i}}, \mathrm{yi}\right)$ corresponds to the coordinates for the position of the i-emmiter [14-16].

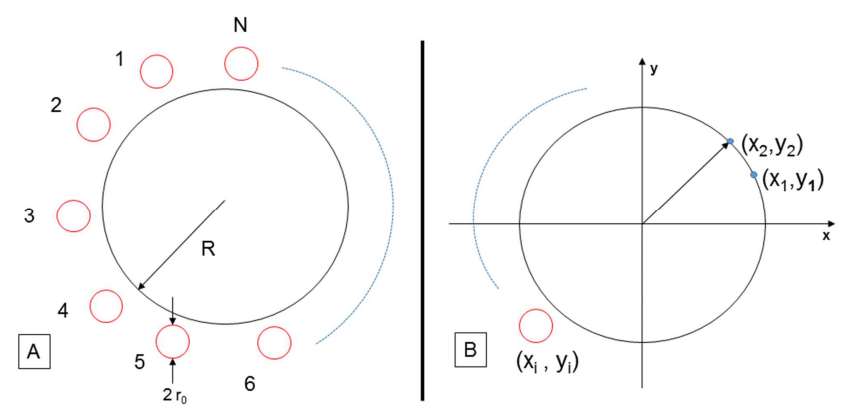

Figure 4. Described the position light; A-) Lamps position and variation of the quantity; $B$-) Lamps coordinated described.

The value for I $(\mathrm{x}, \mathrm{y})$, however given by

$$
I(x, y)=\left(r_{0}\right) I_{0} \sum_{i=1}^{N} \frac{1}{\left(x-x_{i}\right)^{2}+\left(y-y_{i}\right)^{2}}
$$

At the center of the configuration $\mathrm{x}=\mathrm{y}=0$, since $\mathrm{x}^{2}+\mathrm{y}^{2} \sim \mathrm{R}^{2}$ with $\mathrm{R}$ approximation the chamber is radio, $I(0,0)=\frac{r_{0} I_{0} N}{R}$, for analyzing of the intensity variation was calculated the changes in the axis $X$ from 0 until $6 \mathrm{~cm}$ with $y=0$ (Figure 5).

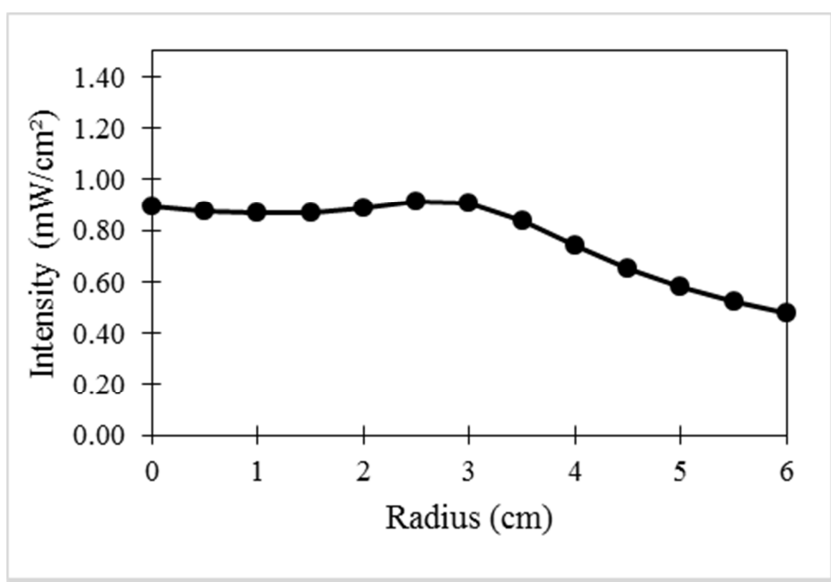

Figure 5. Intensity variation in the axis $X$ with $y=0$ been made the radius.

The results described above show that the UV intensity on the reactor is approximately $0.89 \mathrm{~mW} / \mathrm{cm}^{2}$ and practically not vary until $3 \mathrm{~cm}$ in radius. In this reactor, the light intensity does not decrease due to homogeneity of its axial geometric.

If $\mathrm{r}_{0} / \mathrm{R} \sim 1 / \mathrm{N}$; we can produce at the center at least, the surface intensity. The great advantage is that the overall variation of light intensity with distance from the center is much smaller than in the axial case. This allow a large volume inside the chamber, exposed to practically the equivalent intensity and therefore minimizing the effect of big variations an example of the light intensity distribution for a symmetric 6 emitter distribution is resented in Figure 6. While near the emitters the equal intensity plots resent oscillations compatible with the number of emitters, near the center, the intensity is isotropic and with small radial variation for the overall light intensity [13, 17-19].

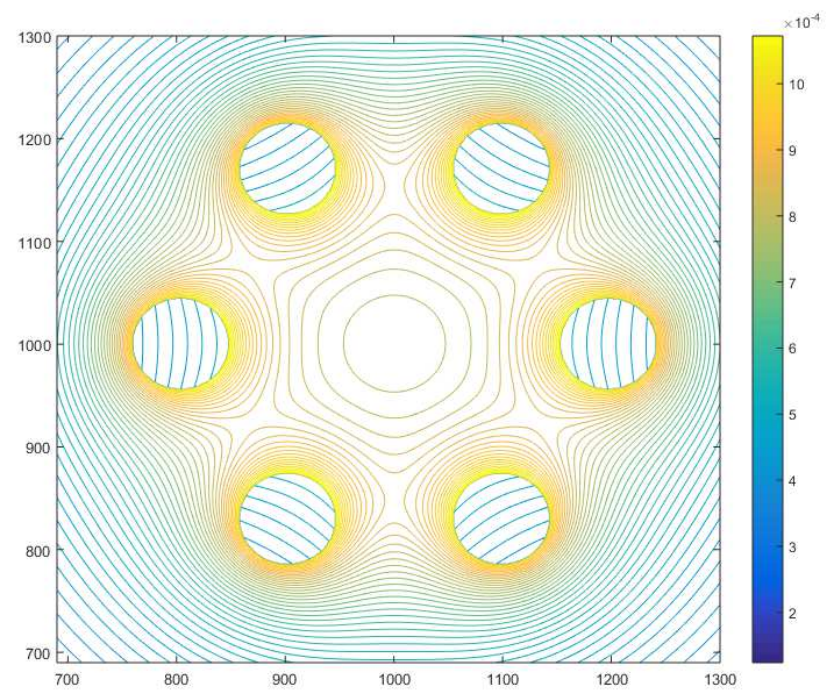

Figure 6. Color maps of the intensity light distribution, which blue line represent different intensity.

If consider $r_{0} / R$ as a relevant geometric factor, when we move $10 \%$ of $\mathrm{R}$ from the center, the intensity variation is 
approximately $0.9 \mathrm{~mW} / \mathrm{cm}^{2}$ per lamp, while moving $50 \%$ of the $\mathrm{R}$, we found $\mathrm{I}=0.95 \times \mathrm{I}_{0}$.

To continuous the quantitative analyzing of the distribution of the light was applied the same simulation in the matlab, however have the focus in the center of the lamps (Figure 7).

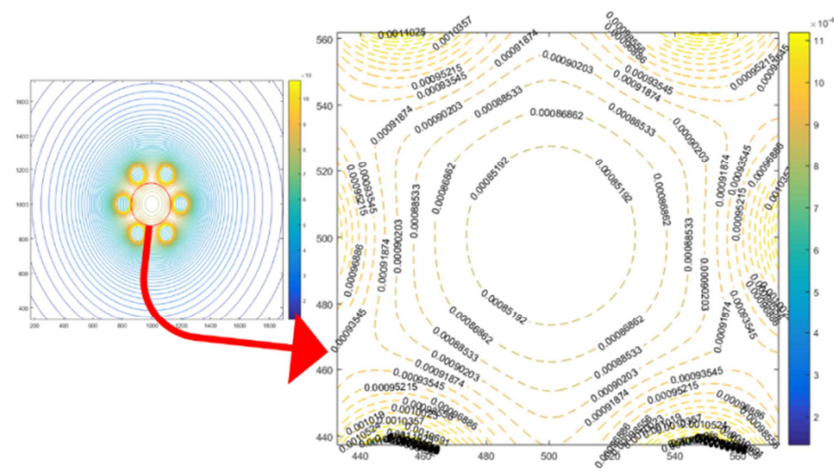

Figure 7. Described the contours maps with 60 lines and de intensity of each point in the reactor and the contours is on region with the same intensity.

It have the quantitative number in the each line of the intensity present on the tube; firstly in the center position have the line that the value is $\sim 0.85 \mathrm{~mW} / \mathrm{cm}^{2}$, when see the next line the value is $\sim 0.87 \mathrm{~mW} / \mathrm{cm}^{2}$. Consequently, the next is $\sim 0.88 \mathrm{~mW} / \mathrm{cm}^{2}$. Is possible understanding that the variation of the intensity is $0.03 \mathrm{~mW} / \mathrm{cm}^{2}$, practically have the homogenous areas, these results provide us with an exceptional indicative for the advantages of our reactor when compared with existent in the market.

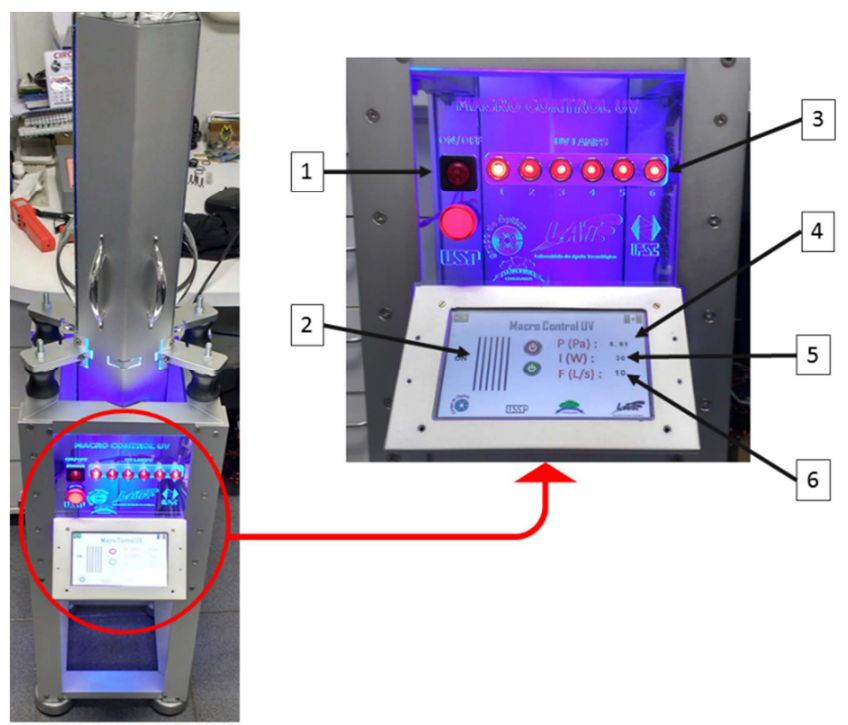

Figure 8. UV-C reactor with details of the panel control; 1-) Bottom on/off; 2-)Image dos the lamps on the reactor; 3-) Sensor that shown the operation of the light; 4-)Sensor of the pressure; 5-) Sensor of the intensity light; 6-) Flowmeter of the flow rate.

The reactor we have constructed is well described in Figure 4. A central quartz tube of diameter $\varnothing=5 \mathrm{~cm}$ is surrounded by six mercury lamps. The lamps are diameter symmetric distributed each lamp emits $10 \mathrm{~W}$ of UV (combined lines) consuming about $40 \mathrm{~W}$ of electrical energy.
The total extension of the tube is around $100 \mathrm{~cm}$. The chamber where fluid is exposed to UV is about $8,000 \mathrm{~cm}^{3}$ (or 8L).

For details, the total electrical power injected in the system is about $250 \mathrm{~W}$, we show the overall device while in figure 8 are schematic allow to point out a few details. The water to be decontaminated flow from down to up in this vertical geometry. Two gate values, allow isolation of the chamber if necessary. An electronic panel control, allow visualization of the operation together with receiving alert if the discharge in each of the lamps shows any problem.

In figure $4 \mathrm{c}$, a horizontal cut of the device is presented. The six lamps have a close contact with the quartz tube having on the most outside part, reflectors to send back to the system.
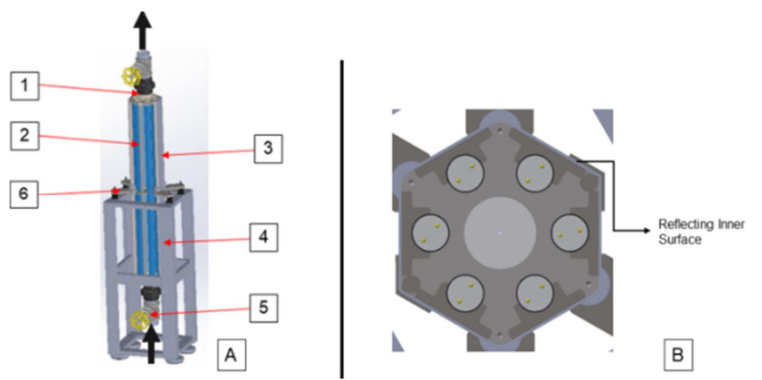

Figure 9. A-) Mechanical design, the black arrow is a direction flow; 1-) Union of the tube with quartz tube; 2-)UV-C lamp with $30 \mathrm{~W}$ the OSRAM; 3-)Aluminum reflector; 4-) Base of structure; 5-) Global valve; $B$-) Shown the geometry design of the lamp surrounding the quartz tube.

Light emitted in the opposite radial direction to the chamber. The measured total intensity at the center of the chamber is $\sim 0.85 \mathrm{~mW} / \mathrm{cm}^{2}$ per lamp. The control allow operating with two, four or six lamps, and therefore we can have $0.85 ; 0.57$ or $0.26 \mathrm{~mW} / \mathrm{cm}^{2}$ of intensity at the chamber center. The total quantity of energy incident and the volume of liquid contained in the chamber is dependent on the total time of expose.

\subsection{Circulating Tank and Decontamination}

The several set up for circulations liquid decontamination is schematically represented in figure 5 .

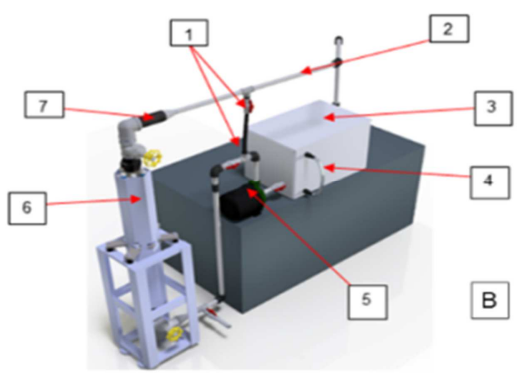

Figure 10. Scaling down Project and experimental setup to validation and testing the decontamination broccoli; 1-) By-pass to control the velocity of water in the reactor; 2-) Pipeline for circulation water on the system; 3-) Tank to washing the vegetal broccoli; 4-) Level gauge; 5-) Pump to circulation water in the system; 6-) UV reactor and components; 7-) Tube connections of system with reducing adaptors 2 " to $1 / 2$ ". 
Considering the reactor volume as $V_{R}$ and the tank volume $\mathrm{V}_{\mathrm{T}}$, the total volume circulating is $\mathrm{V}=\mathrm{V}_{\mathrm{T}}+\mathrm{V}_{\mathrm{R}}$. Having a liquid flow rate, produced by the pump, of $\mathrm{Q}$ (volume/time), the liquid fraction constantly exposed to UV is $\frac{V_{R}}{V_{R}}+V_{T}$.

The time of permanence for the liquid stay exposed to UV is $\frac{V_{R}}{Q}$ and therefore the necessary time to expose to UV the whole volume is the system is $\Delta t_{t}=V_{R}+\frac{V_{T}}{Q}$ higher flow rate demands less time for a fall exposition to the whole volume to UV. Therefore, if the system circulate during a time interval $\Delta \mathrm{t}$, the total exposure is proportional to $\Delta \mathrm{t}$ and therefore proportional to $\frac{\Delta t}{\Delta t_{t}}$ and therefore proportional to $\frac{Q}{V_{R}}+V_{T}$. The overall dose of energy delivered to the solution will also be proportional to this quantity and so will be the total dose (D).

Therefore, expect of total microbial decrease to increase with the flow rate, therefore, since the microbiological killing by UV depends on the dose, will also depends on the flow rate [20].

The first performed experiment consisted of in producing a standard contamination of the water and measuring the decreasing of the microbial population as a function of time. We have contaminated the overall liquid with $10^{5} \mathrm{UFC} / \mathrm{mL}$ of E. coli operating with a flow rate of $80 \mathrm{~L} / \mathrm{min}$ in a total volume of $30 \mathrm{~L}$ and six lamps in the reactor the collection of solution samples in intervals of 25 min producing the result indicated in Figure 6 [18, 21].

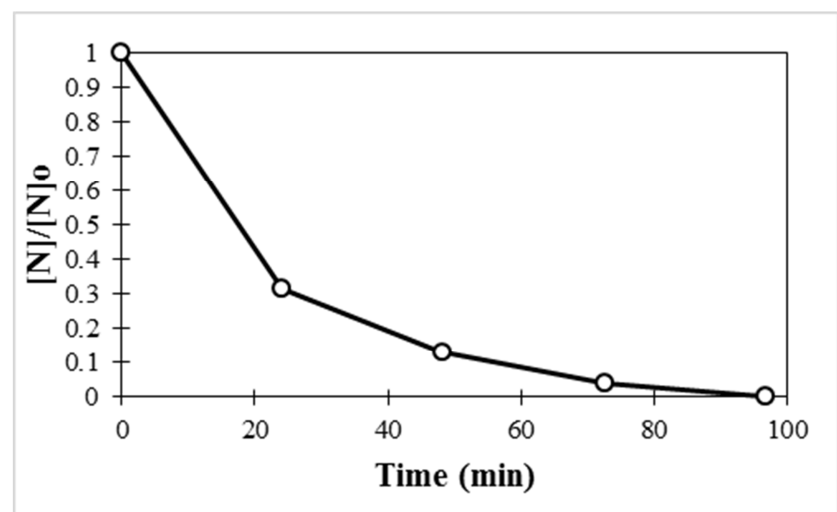

Figure 11. Inactivation ratio for E. coli with initial concentration $10^{5}$ UFC/mL and flow rate $80 \mathrm{~L} / \mathrm{min}$.

The results of Figure 6 characterize the capacity to decontamination by assembled system. Defining as $\gamma$ the probability of destroying a microorganism per unit of time when the solution is circulation on the system, a simple kinetic equation can be build resulting in [22]:

$$
\frac{d N}{d t}=-\gamma \times N
$$

Resulting in the traditional exponential low for the decreasing number of microorganism, $\mathrm{N}=\mathrm{N}_{0} \mathrm{e}^{-\mathrm{Yt}}$ the value of $\gamma$ will depend on the flow rate in the system and the overall delivered power $\left(\mathrm{P}_{0}\right)[6,22,23]$.

For the above example presented in the figure $6, \gamma \sim 0.1$ $\min ^{-1}$, followings this simple model. As we have discussed before, increasing the flow, will result in faster inactivation kinetic.

We have however performed experiments varying the flow rate of the system, still using $E$. coli and inactivation kinetic is presented in figure 7.

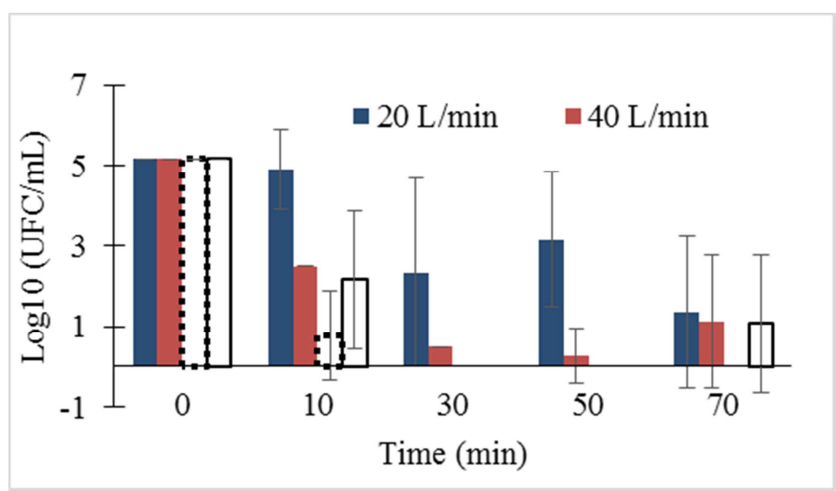

Figure 12. Decontamination of the water in different rate flow, the microorganism is E. coli with $10^{7} \mathrm{UFC} / \mathrm{mL}$ the initial concentration.

As the flow rate is doubled, in the log scale of Figure 7 was observed the line slope of the decrease for microorganism concentration to double as well equivalently if we triple the flow rate the slope also triple, proving the proportionality discussed before. For the $E$. coli used in this experiment we could stablish a relation between $\gamma$ and the flow rate. If for 20 $\mathrm{L} / \mathrm{s}$ we obtain $\gamma \sim 0.04 \mathrm{~min}^{-1}$, for a flow rate (Q).

$$
\gamma=0.04 \mathrm{Q} / 20
$$

That is certainly in close agreement with the experiment.

\section{Decontamination of Broccoli with the Circulating System}

After demonstrating the capacity of the system in inactivating the microorganism, we want now to add in the tank-contaminated broccoli, and through the circulation, demonstrate the overall decontamination of the target. The overall schematic for this experiment is in Figure 8.

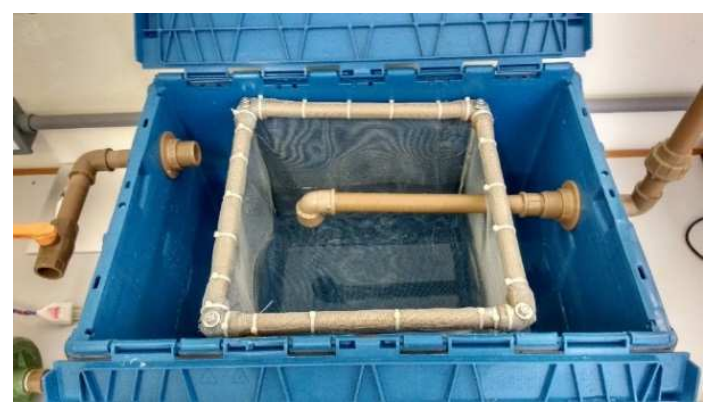

Figure 13. Photographic of the schematic experiment of the washing broccoli.

The fluid, water, is forced to flow by the pump at a flow rate Q. After passing to UV exposition, the water is returned to the tank directly to cage containing parts of the broccoli. 
During the circulation, the water supposed to remove the microorganism from the target broccoli and retain in solution to be exposed to UV with the final inactivation. The dynamics now is quite different from the last experiment where an initial concentration of microorganism were inoculated into the solution and the system could act and its.

The new situation has now a source of microorganism that constantly load the solution while the UV exposition try to eliminate it. The overall equation for the kinetic of inactivation must have now extra terms to be considered.

$$
\frac{d N}{d t}=-\gamma * N+F(N, t)
$$

Where $\mathrm{F}$ represents a function of microorganism injection rate into the solution, recontamination by the solution (while still having microorganism). The function $\mathrm{F}$, must depend on many facts involving the capacity of the circulating fluid to remove the target contaminants by itself. To allow a better understand of this kinetic, we have performed an experiment where broccolis were contaminated with a solution containing $10^{7} \mathrm{UFC} / \mathrm{mL}$ by immersion during a time of 70 min. After this contaminating period, the contaminated peace were inserted into the system and put to circulate. Periodically samples of the solution and broccoli were collected and analyzed concerning a contain of microorganisms. The overall result is presented in Figure 9.

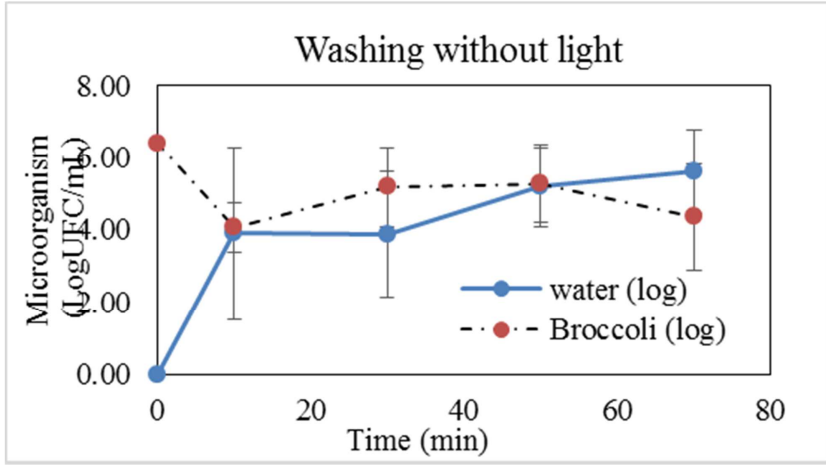

Figure 14. Analyze of the leaching of broccoli in the water without light.

The concentration of microorganisms in the solution is increasing with time, while the concentration in the broccoli is decreasing with time colling $\mathrm{N}_{\mathrm{s}}$ the concentration in solution and $\mathrm{N}_{\mathrm{B}}$ the concentration in broccoli, we must have, in first approximation $\mathrm{N}_{\mathrm{S}}+\mathrm{N}_{\mathrm{B}}$ constant (if no multiplication is taking place). In that case (Figure 15).

$$
\frac{d N_{S}}{d t}=-\frac{d N_{B}}{d t}
$$

In this situation, eventually both get to be zero, when the rate of remoting of microorganism gets equal to the rate of recontamination looking into the solution, $\gamma=0$ and $\frac{d N_{s}}{d t}=F(N, t)$ corresponds to the represented points. It is a function with a fast growth at the beginning followed by a tendency to saturation. We must have the equilibration when $\mathrm{N}_{\mathrm{S}}=\mathrm{N}_{\mathrm{B}}$, because in such case the remove of contaminants from the broccoli by the solution loses the efficacy, and an equilibrium is established. The results of Figure 9 provide us with indication that the system is actually in such equilibrium.

The next experiment is the addition of the inactivation mechanism placing $\gamma \neq 0$ but turning on the lights on the main chamber. Equivalent to the experiment with no light, about $10^{7} \mathrm{UFC} / \mathrm{mL}$ were used to contaminate the broccoli, which was placed on the cage and water circulation with UV inactivation start. For this experiment we have $\mathrm{Q}=80 \mathrm{~L} / \mathrm{min}$.

Again, samples of the water well as from the broccoli was examined periodically and the concentration of $E$. coli evaluated through the colony forming units (CFU) of the microorganism. The obtained result is displayed in figure 10 .

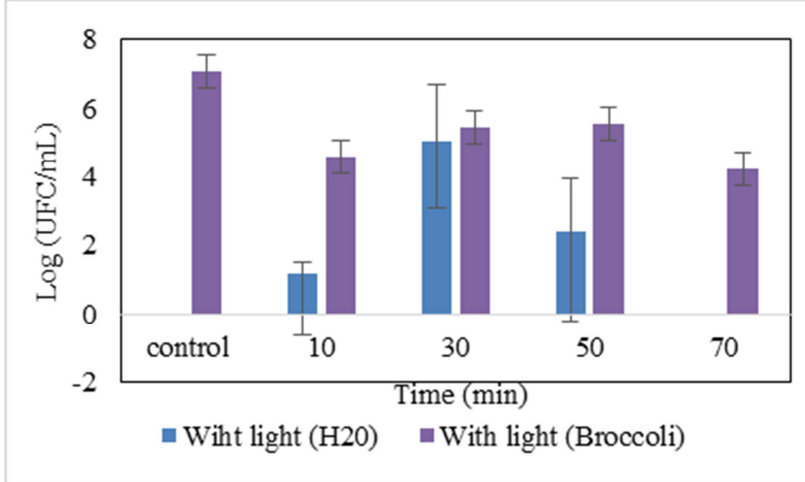

Figure 16. Analyze of the leaching of broccoli in the water with UV-C light.

While the concentration of microorganisms in the broccoli presents an overall, decrease with time, demonstrations a remoting of microorganism that overcome the accumulation of them. The amount of microorganism in solution revels a fully different behavior when compared with no-light mode.

For the solution component, in a first stage the numbers of microorganism increase, showing a dissolution of microorganism frequently in the broccoli quite fast. The number in solution researches a maximum and start to decrease. It only solution is observed.

$$
\frac{d N_{S}}{d t}=L\left(N_{B}, t\right)-\gamma N_{S}
$$

Where $L\left(N_{B}, t\right)$ a loading rate is depends on the quantity in the broccolis and the time. It is reasonable to consider $L\left(N_{B}, t\right)=\alpha\left(N_{B}-N_{S}\right)$ proportional to the concentration in the broccolis and a rate constant $\alpha$. On the other hand, the number of microorganisms in the broccoli must decreases, in a first approximation as

$$
\frac{d N_{B}}{d t}=-\beta\left(N_{B}-N_{S}\right)
$$

Indicating that while $\mathrm{N}_{\mathrm{B}}>\mathrm{N}_{\mathrm{S}}$ there will be transference from broccoli to solution. Because of such considerations, we have two coupled differential equations

$$
\begin{aligned}
& \frac{d N_{S}}{d t}=\alpha\left(N_{B}-N_{S}\right)-\gamma N_{S} \\
& \frac{d N_{B}}{d t}=-\beta\left(N_{B}-N_{S}\right)
\end{aligned}
$$

When $\mathrm{N}_{\mathrm{B}} \sim \mathrm{N}$ and $\mathrm{N}_{\mathrm{S}}=0$, the number in solution is 
increasing because $\frac{d N_{S}}{d t}>0$. Reaching the point where $\frac{d N_{S}}{d t}=0$, when both terms become equal $\alpha\left(N_{B}-N_{S}\right)=$ $\gamma N_{S}$ as $N_{S}=\frac{\alpha}{\alpha+\gamma} N_{B}$. From that point on, $\frac{d N_{S}}{d t}<0$ and $N_{S}$ is now decreasing with time. The solution for $\mathrm{N}_{B}$ also decreases as much as $\mathrm{N}_{\mathrm{B}}>\mathrm{N}_{\mathrm{S}}$. But considering $\alpha, \gamma>0, \mathrm{~N}_{\mathrm{S}}$ will be at maximum $N_{S}=\frac{\alpha}{\alpha+\gamma} N_{B}<N_{B}$. Therefore $N_{B}$ will always be a decreasing function with time. The complete solution an be obtained if the coupled equations are solved. The obtained result, as presented in Figure 10 is in good quantitative agreement with the obtained results.

\section{Conclusion}

The intensity of UVC light distributed in the internal part of the reactor is practically homogeneous due to the developed geometry. The kinetics of microbial death presented no great influence on this variation. That is, any volume of water contained in the process can be decontaminated.

This behavior cannot be compared to reactors with little axial geometry and centralized lamp. The decay is exponential in relation to distance, that is for high volume and speed, an increase in the diameter of the outer tube compared to the inner tube is required.

\section{Acknowledgements}

The authors acknowledge the support provided by FAPESP (São Paulo Research Foundation), grant number 2013/07276-1 (CEPOF-CEPID Program).

\section{References}

[1] FDA. Kinetics of microbial inactivation for alternative food processing technologies - Ultraviolet leght. 2017.

[2] Crook, J. A., Rossitto, P. V, Parko, J., Koutchma, T. \& Cullor, J. S. Efficacy of ultraviolet (UV-C) light in a thin-film turbulent flow for the reduction of milkborne pathogens. Foodborne Pathog. Dis. 12, 506-513 (2015).

[3] Manzocco, L. et al. Surface decontamination of fresh-cut apple by UV-C light exposure: Effects on structure, colour and sensory properties. Postharvest Biol. Technol. 61, 165-171 (2011).

[4] Bialka, K. L. \& Demirci, A. Efficacy of pulsed UV-light for the decontamination of Escherichia coli O157: H7 and Salmonella spp. on raspberries and strawberries. J. Food Sci. 73, M201-M207 (2008).

[5] Gomez-Lopez, V. M., Ragaert, P., Debevere, J. \& Devlieghere, F. Pulsed light for food decontamination: a review. Trends food Sci. Technol. 18, 464-473 (2007).

[6] Ye, Z., Koutchma, T., Parisi, B., Larkin, J. \& Forney, L. J. Ultraviolet inactivation kinetics of Escherichia coli and Yersinia pseudotuberculosis in annular reactors. J. Food Sci. 72, E271-E278 (2007).
[7] Selma, M. V, Allende, A., López-Gálvez, F., Conesa, M. A. \& Gil, M. I. Disinfection potential of ozone, ultraviolet-C and their combination in wash water for the fresh-cut vegetable industry. Food Microbiol. 25, 809-814 (2008).

[8] Harris, R. F. Effect of water potential on microbial growth and activity. Water potential relations soil Microbiol. 23-95 (1981).

[9] Santos, E. R. et al. Reator de UV-Ozônio com lâmpada a vapor de mercúrio a alta pressão modificada para tratamento superficial de óxidos transparentes condutivos utilizados em dispositivos poliméricos eletroluminescentes. Quim. Nov. 33, 1779-1783 (2010).

[10] Crittenden, J. C., Hu, S., Hand, D. W. \& Green, S. A. A kinetic model for $\mathrm{H} 2 \mathrm{O} 2 / \mathrm{UV}$ process in a completely mixed batch reactor. Water Res. 33, 2315-2328 (1999).

[11] Guerrero-Beltr· n, J. A. \& Barbosa-C · novas, G. V. Advantages and limitations on processing foods by UV light. Rev. Agaroquimica y Tecnol. Aliment. 10, 137-147 (2004).

[12] Severin, B. F., Suidan, M. T., Rittmann, B. E. \& Engelbrecht, R. $\mathrm{S}$. Inactivation kinetics in a flow-through UV reactor. J. (Water Pollut. Control Fed. 164-169 (1984).

[13] Cassano, A. E., Martin, C. A., Brandi, R. J. \& Alfano, O. M. Photoreactor analysis and design: fundamentals and applications. Ind. Eng. Chem. Res. 34, 2155-2201 (1995).

[14] Oguma, K., Kita, R. \& Takizawa, S. Effects of Arrangement of UV Light-Emitting Diodes on the Inactivation Efficiency of Microorganisms in Water. Photochem. Photobiol. (2016).

[15] Oguma, K., Rattanakul, S. \& Bolton, J. R. Application of UV Light-Emitting Diodes to adenovirus in water. J. Environ. Eng. 142, 4015082 (2015).

[16] Matak, K. Effects of UV irradiation on the reduction of bacterial pathogens and chemical indicators of milk. (2004).

[17] Ye, Z. UV disinfection between concentric cylinders. (Georgia Institute of Technology, 2007).

[18] Merchuk, J. C., Garcia-Camacho, F. \& Molina-Grima, E. Photobioreactor design and fluid dynamics. Chem. Biochem. Eng. Q. 21, 345-355 (2007).

[19] Kowalski, W. J. Design and optimization of UVGI air disinfection systems. (2001).

[20] Fredericks, I. N., du Toit, M. \& Krügel, M. Efficacy of ultraviolet radiation as an alternative technology to inactivate microorganisms in grape juices and wines. Food Microbiol. 28, 510-517 (2011).

[21] Geralde, M. C. et al. Pneumonia treatment by photodynamic therapy with extracorporeal illumination-an experimental model. Physiol. Rep. 5, e13190 (2017).

[22] Koutchma, T. Advances in ultraviolet light technology for non-thermal processing of liquid foods. Food Bioprocess Technol. 2, 138-155 (2009).

[23] Silva, A. B., Lima Filho, N. M., Palha, M. A. P. F. \& Sarmento, S. M. Kinetics of water disinfection using UV-C radiation. Fuel $110,114-123$ (2013). 